\title{
Taquicardia ventricular por reentrada rama - rama en pacientes con Enfermedad de Steinert: Serie de tres casos y revisión de la literatura.
}

Rodrigo Isa, Mauricio Moreno, Jorge Palazzolo.

Hospital El Carmen de Maipú y Clínica Redsalud Vitacura, Santiago de Chile.

Resumen: Se presentan tres casos clínicos de pacientes con en Enfermedad de Steinert y Taquicardia ventricular recurrente asociada. En los 3 casos el diagnóstico involucró un exhaustivo estudio electrofisiológico que demostró que se trataban de $\mathrm{TV}$ rama a rama. Se describen los mecanismos y las maniobras electrofisiológicas para establecer el diagnóstico, como también el tratamiento. Incluye una extensa revisión bibliográfica.

Palabras clave: taquicardia ventricular rama a rama; Steinert, enfermedad de; ablación; estudio electrofisiológico.

\section{Bundle branch reentrant ventricular tachycardia in patients with Steinert's disease: Report of three cases and review of the literature}

This is a report of three patients with Steinert's disease who presented with ventricular tachycardia requiring electrical cardioversion. Extensive electrophysiologic study demonstrated an underlying bundle branch ventricular tachycardia. The mechanisms and the electrophysiological approach to diagnosis are described in detail and the treatment selected is discussed. An extensive review of the literature is included.

Keywords: ventricular tachycardia, bundle branch reentrant; Steinert's disease; electrophysiology; ablation. 


\section{Introducción:}

Los pacientes que sufren enfermedades neuromusculares tienen, con frecuencia, diferentes manifestaciones cardiovasculares ${ }^{1}$.

En este artículo se describen 3 casos clínicos de pacientes consecutivos que se presentaron con arritmias ventriculares y enfermedad de Steinert. Se revisa la asociación con la enfermedad neuromuscular de base y se discuten los mecanismos electrofisiológicos.

\section{Caso clínico $\mathbf{N}^{\mathbf{0}} \mathbf{1}$ :}

Varón de 38 años con diagnóstico de distrofia miotónica de Steinert. Ingresa al servicio de urgencias tras haber sido reanimado en su domicilio de varios episodios de taquicardia de QRS ancho sincopal. Se realizó CVE efectiva en cada episodio retomando ritmo sinusal con bloqueo auriculoventricular de primer grado y bloqueo completo de rama izquierda.

Ingresado en servicio de urgencia repite episodios de igual taquicardia con compromiso hemodinámico, requiriendo cardioversión eléctrica (CVE).

El ecocardiograma mostró la presencia de una miocardiopatía dilatada con hipokinesia difusa, FEVI de 30\% y sin defectos valvulares significativos.

Una vez obtenido el consentimiento informado se realizó un estudio electrofisiológico convencional: punción venosa femoral derecha triple con introductores $7 \mathrm{fr}$. Catéter decapolar en seno coronario, catéter cuadripolar en His y otro en ventrículo derecho. Se obtiene registro intracavitario en ritmo sinusal con intervalos basales

Fig 1 A y B.

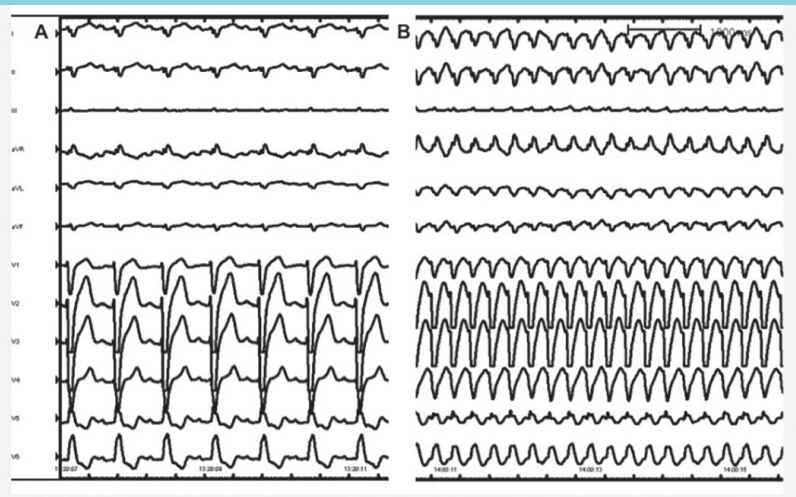

ECG de 12 derivaciones en ritmo sinusal (A) y en TV (B) del paciente $N^{\circ} 1$. En el primero se observa ritmo sinusal con $B A V$ de $1^{\circ}$ grado y BCRI. En el segundo, una Taquicardia regular de QRS ancho e imagen de BCRI. Nótese la similitud en morfología y eje eléctrico entre el RS y la TV, característica de este tipo de taquicardia (explicación en texto).

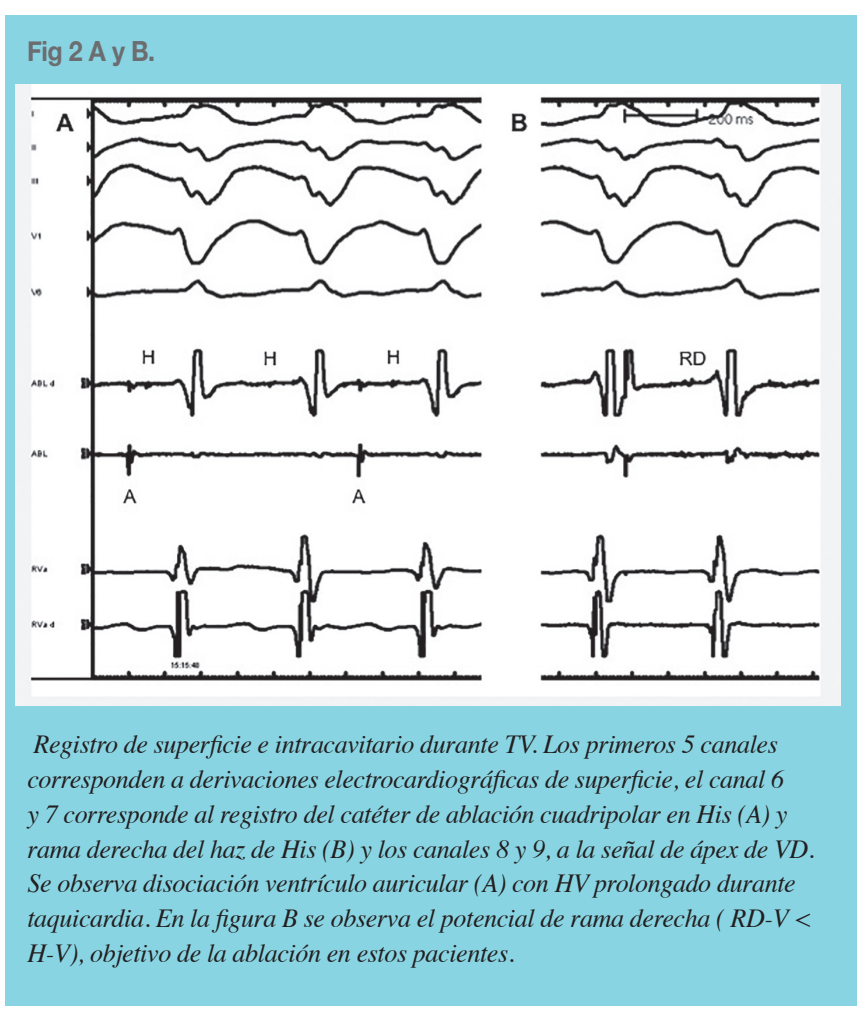

alterados (AH: $80 \mathrm{~ms}$ y HV: $85 \mathrm{~ms}$ ). La estimulación ventricular desde el ápex de VD mostró ausencia de conducción ventrículo - auricular. Con estimulación programada desde VD con un extraestímulo (600 / 340 $\mathrm{ms})$ se induce una taquicardia regular de QRS ancho con imagen de BCRI idéntica a la taquicardia clínica registrada y similar a la morfología del QRS en ritmo sinusal con ciclo de 340 ms (Fig 1A y B), disociación ventrículo auricular y electrograma hisiano precediendo cada señal ventricular con intervalo HV de $90 \mathrm{~ms}$ (Fig $2 \mathrm{~A}$ y B). El encarrilamiento desde el ápex del ventrículo derecho mostró una respuesta $\mathrm{V}-\mathrm{H}-\mathrm{V}$ con fusión discreta e intervalo post estimulación 25 ms mayor que el ciclo de la taquicardia. Todos estos hallazgos confirman el diagnóstico de una Taquicardia ventricular por reentrada rama- rama tipo 1 . Se interrumpió la taquicardia con sobre estimulación ventricular y, dado el riesgo de bloqueo AV completo que conlleva la ablación de este tipo de taquicardia y el grado severo de disfunción ventricular, se decide diferir ésta hasta una vez implantado un desfibrilador.

Se implantó un desfibrilador bicameral Biotronik Intica 5 DR-T (DAI DDD) en región subclavia izquierda con electrodo "monocoil" en la zona medio septal del VD y se programó la ablación de TV una semana después de 


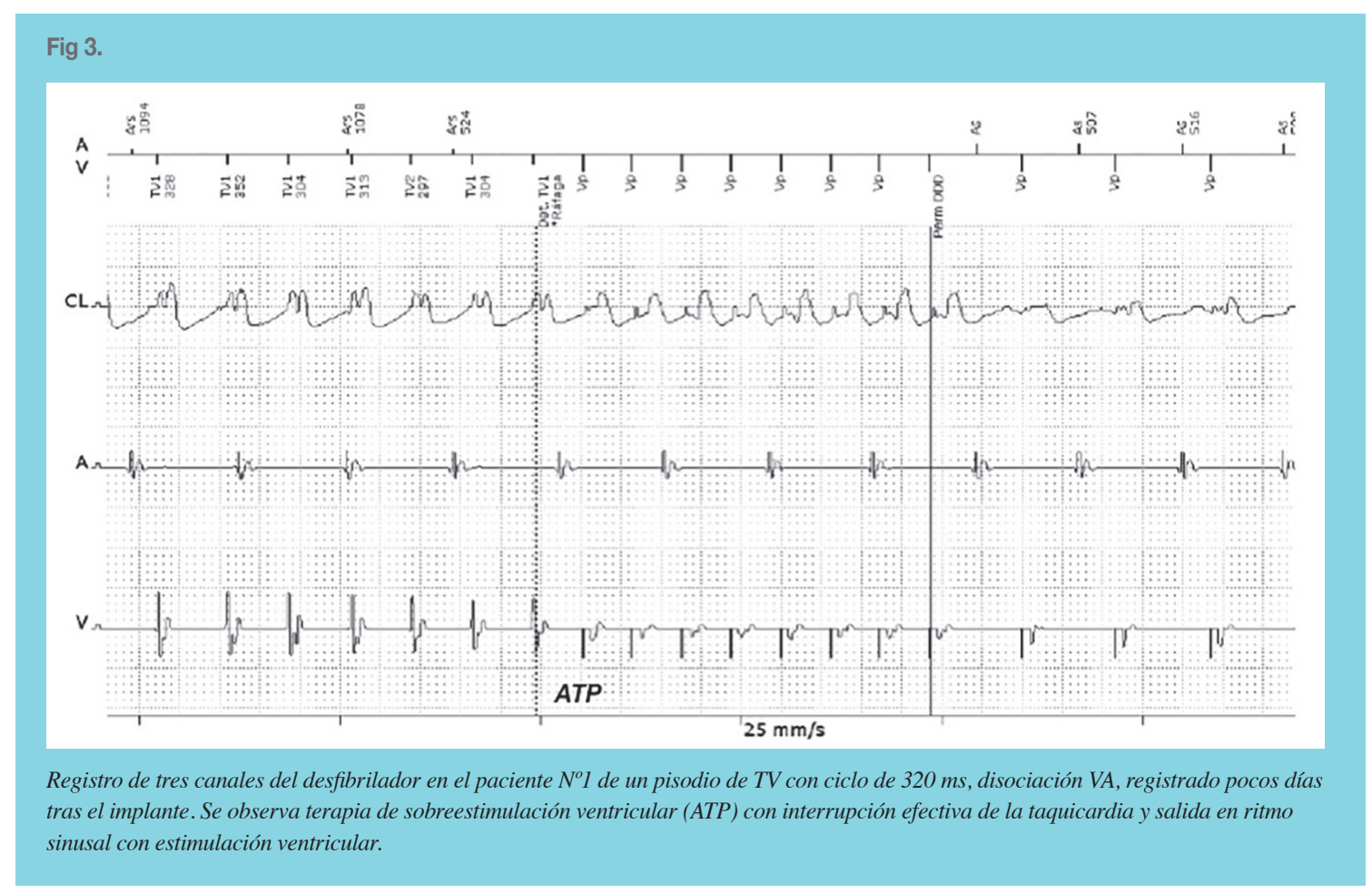

implantado.

Se revisó el DAI DDD que mostró 2 episodios de TV con disociación VA y ciclo de 320 ms tratados con sobre estimulación ventricular (ATP) de forma exitosa (Fig 3). Se desactivan las detecciones, se realizó una induc-

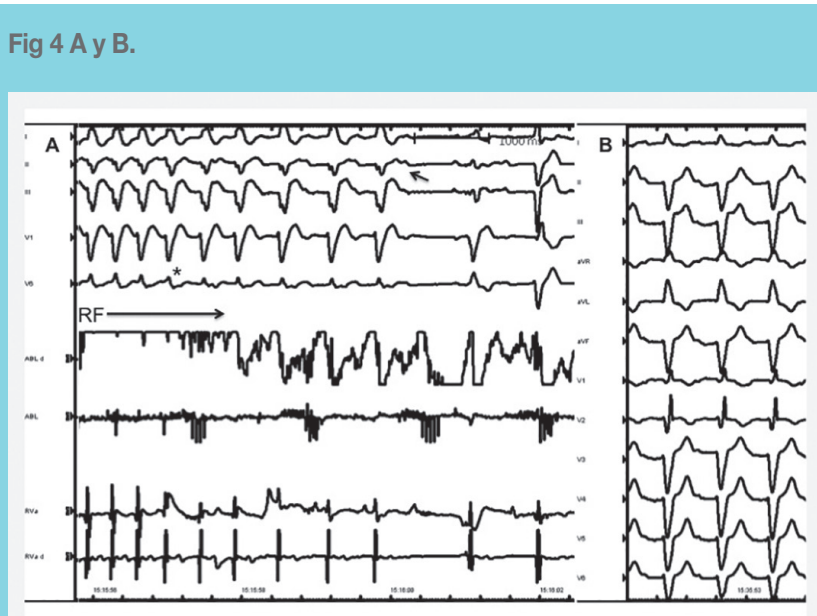

A. Registro de superficie e intracavitario de TV $(25 \mathrm{~mm} / \mathrm{s})$ durante ablación con $R F(R F)$. Se observa interrupción brusca de la TV en el $4^{\circ}$ latido $\left(^{*}\right)$, seguido de taquicardia sinusal conducida con BAV de $2^{\circ}$ Grado de tipo Wenckebach (flecha indica P bloqueada), con latido siguiente conducido con PR más corto y BCRI y el subsiguiente con PR largo y BCRD consiguiendo el objetivo en la ablación de la TVRR: BCRD, como se muestra en B (ECG de 12 derivaciones post ablación con $R F$ ). ción de TV con las mismas características ya descritas y se realizó una ablación con radiofrecuencia con catéter de $4 \mathrm{~mm}\left(50 \mathrm{w} / 65^{\circ}\right)$ en la zona de la rama derecha proximal del haz de His con interrupción de la taquicardia a los 4 segundos y retorno a ritmo sinusal con BCRD (Fig 4A y B).

El procedimiento no presentó complicaciones y el paciente fue egresado a las 72 horas sin observarse recurrencia de $\mathrm{TV}$ ni reconducción de la rama derecha del Haz de His.

Asistió a control a los 2 meses del procedimiento por sentir palpitaciones. La revisión del DAI DDD mostró 3 episodios de Fibrilación auricular auto limitados y 4 episodios de taquicardia regular con ciclo de $370 \mathrm{~ms}$ y asociación VA $=1: 1$ interpretados por el dispositivo como taquicardia ventricular y tratándolos con sobre estimulación ventricular, que consiguió evidenciar disociación ventrículo auricular y enlentecimiento del ciclo ventricular posterior a la estimulación sin modificación del ciclo auricular, confirmándose el diagnóstico de taquicardia auricular con terapia inapropiada por el desfibrilador (Fig 5), por lo que se inició tratamiento antiarrítmico con amiodarona.

En el seguimiento alejado a 12 meses de la ablación no ha presentado nuevos episodios de arritmias auriculares ni ventriculares. 


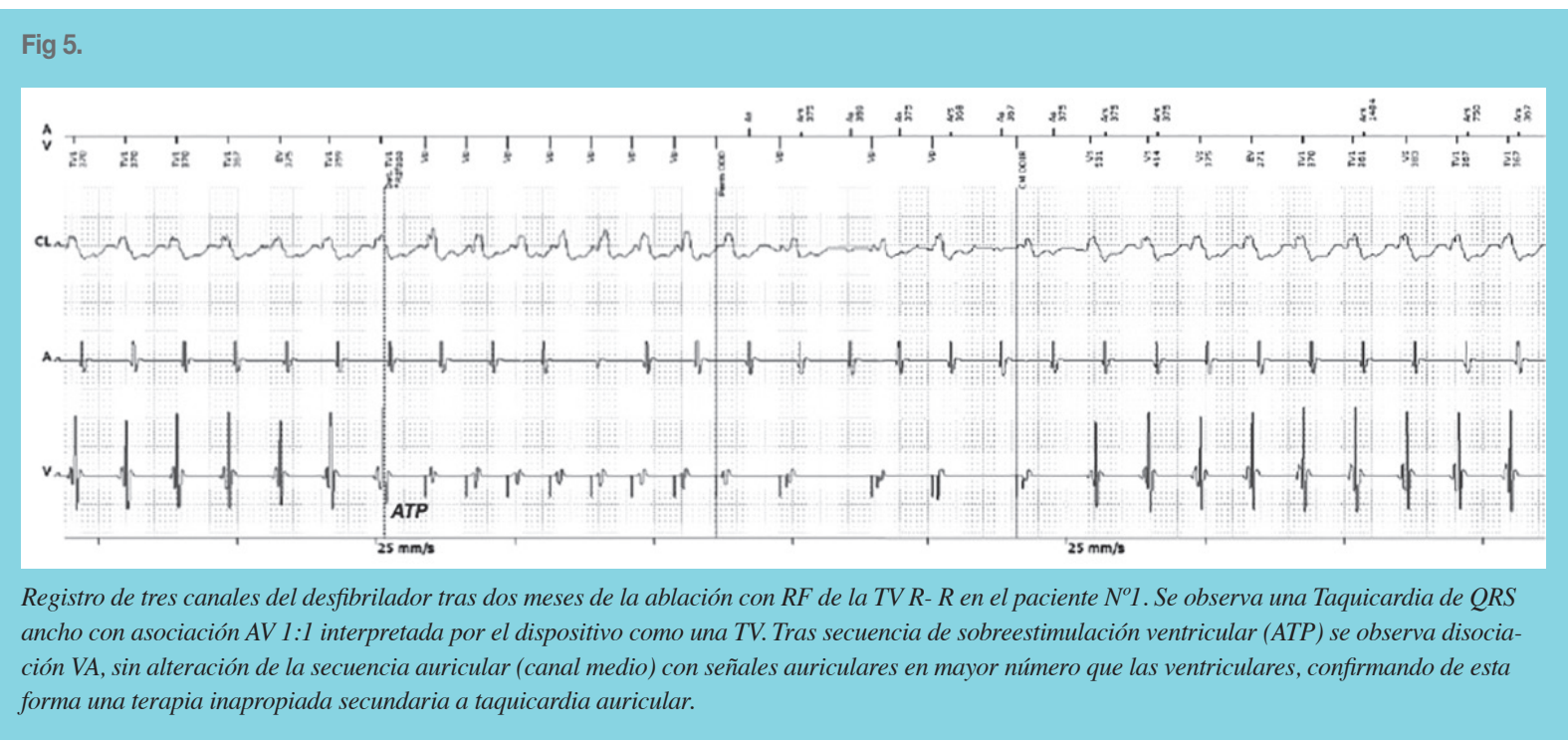

Fig 6 A y B.
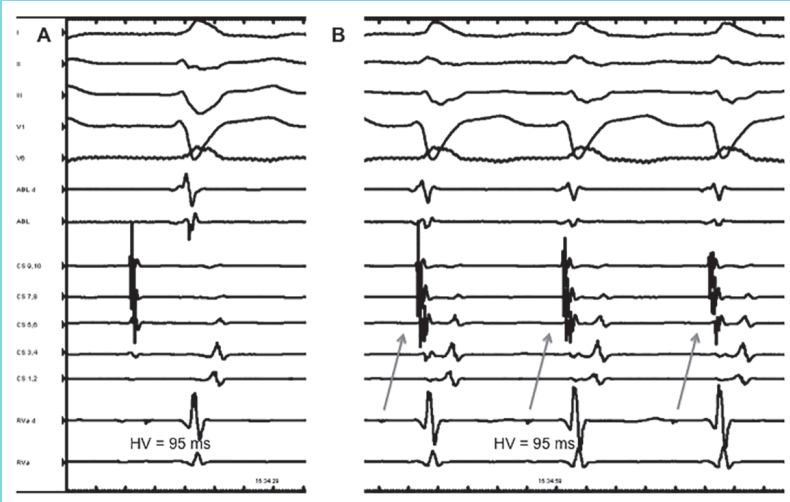

Registro de superficie e intracavitario durante ritmo sinusal (A) y TV (B) del paciente $N^{\circ}$. Los cinco primeros canales corresponden a derivaciones electrocardiográficas de superficie, canales 6 y 7 al registro de ápex de VD, 8 al 12 al seno coronario proximal a distal y 13 y 14 al registro del haz de His. Se observa un HV muy prolongado con BCRI durante RS y TV. Durante TV la relación $V A$ es 1:1 (flechas) y destaca una discreta diferencia en la morfología del QRS entre el RS y la TV (explicación en texto).

\section{Caso clínico $\mathrm{N}^{\circ}$ 2:}

Varón de 58 años, portador de distrofia miotónica de Steinert, con antecedente de 2 hermanos con la misma enfermedad y fallecidos de muerte súbita. Ingresó al servicio de urgencias tras haber presentado varios episodios sincopales en su domicilio. El ECG mostró ritmo sinusal con BAV $1^{\circ}$ grado y BCRI. Un ecocardiograma mostró un ventrículo izquierdo dilatado con diámetro diastólico $70 \mathrm{~mm}$ y FEVI de 30\%. Se realizó el estudio electrofisiológico con el mismo protocolo utilizado en

\section{Fig 7 A y B}

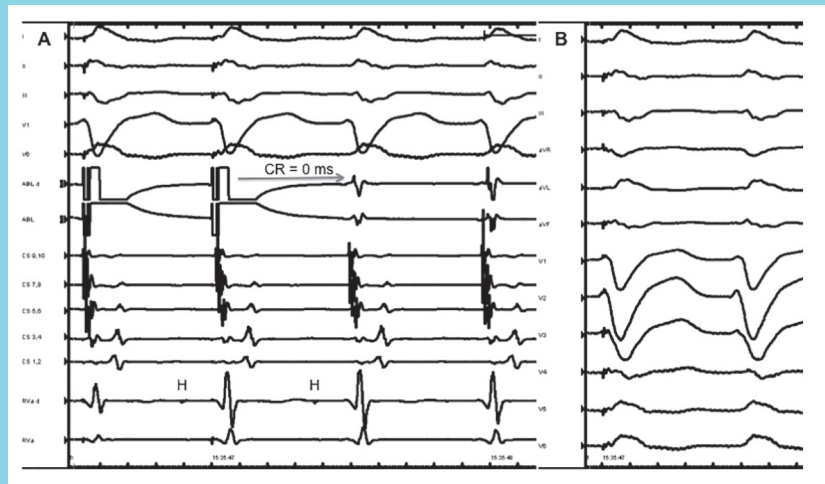

A. Maniobra de encarrilamiento desde ápex de VD durante TV en paciente $N^{\circ} 2$. Mismo orden que registro anterior. Se observa un intervalo post estimulación igual al ciclo de la taquicardia ( $C R=0 \mathrm{~ms}$ )

B. ECG de 12 derivaciones mostrando el último latido del ciclo de estimulación durante TV y el primer latido de retorno de la taquicardia. Se observa fusión oculta ( QRS estimulado idéntico al de la TV).

el caso anterior. Durante estimulación ventricular continua con ciclo de $450 \mathrm{~ms}$ se indujo una taquicardia de QRS ancho con imagen de BCRI, ciclo de $470 \mathrm{~ms}, \mathrm{HV}$ durante taquicardia de 95 ms y conducción VA 1:1 (Fig 6 A y B). El encarrilamiento desde el ápex de VD mostró un ciclo de retorno de $0 \mathrm{~ms}$ y fusión oculta (Fig 7 A y B). La sobreestimulación auricular durante taquicardia, con encarrilamiento de ésta no mostró fusión. Se realizó ablación con radiofrecuencia $\left(50 \mathrm{w} / 65^{\circ}\right)$ sobre la rama derecha proximal con interrupción de la taquicardia de 


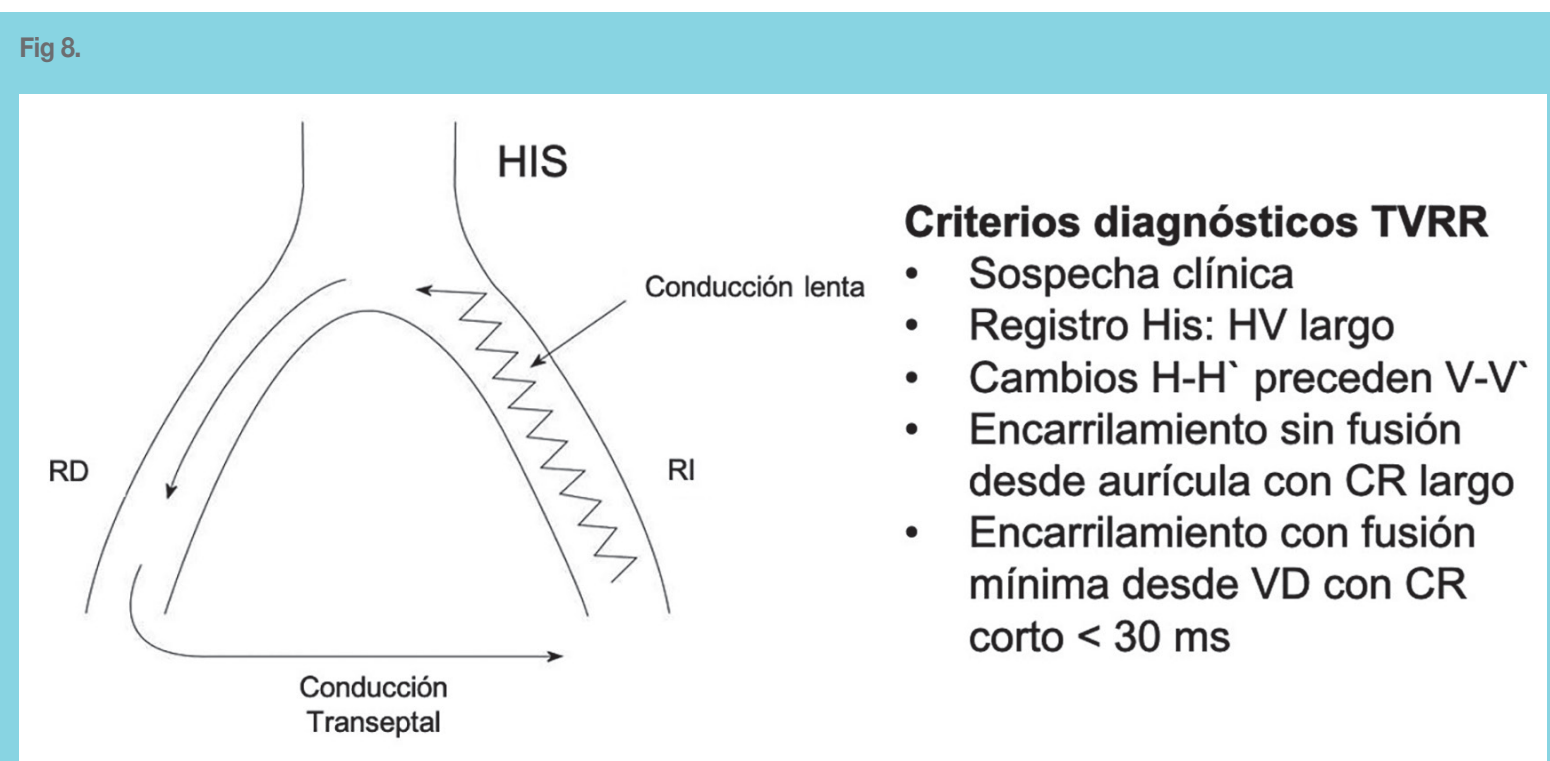

Esquema del mecanismo de la TVRR tipo 1 y sus principales criterios diagnósticos. Ver texto para detalles.

manera inmediata $(<5$ segundos) y apareció un bloqueo AV completo persistente luego de 1 hora de observación por lo que se implantó un desfibrilador VDD Biotronik Rivacor 5 VR-DX DF4 en el mismo acto quirúrgico. Durante un seguimiento de 5 meses no presentó nuevo episodio sincopal ni arritmias ventriculares/auriculares registradas en el dispositivo. El paciente falleció a los 8 meses de implantado el DAI por una neumonía aspirativa.

\section{Caso clínico $\mathrm{N}^{\circ} 3$ :}

Varón de 46 años, con Enfermedad de Steinert, portador de un Desfibrilador Bicameral Biotronik Iperia 5 VR-T DX implantado el 2018 luego de ser reanimado de un episodio de Taquicardia ventricular sincopal. Se presentó 8 meses después en el servicio de urgencia tras haber recibido una descarga del desfibrilador. La interrogación del dispositivo reveló 35 episodios de taquicardia ventricular tratados con sobreestimulación efectiva y un episodio refractario a ATP x 3, que se resolvió tras una descarga de 35 J. El ECG basal mostró RS con BAV de $1^{\circ}$ grado y BCRI. El estudio electrofisiológico indujo con facilidad una taquicardia de QRS ancho con imagen de BCRI y ciclo de 300 ms., disociación ventrículo auricular y registro de His precediendo al QRS por $90 \mathrm{~ms}$. Se efectuaron las maniobras de estimulación ya descritas, que fueron compatibles con el diagnóstico de TV rama - rama tipo 1. Se realizó ablación con RF sobre la rama derecha del haz de His con interrupción de la taquicardia y aparición de BCRD. Sin arritmias durante un seguimiento de 10 meses.

\section{Discusión}

Se presentan 3 pacientes con la misma enfermedad muscular (Distrofia miotónica de Steinert) y manifestaciones cardiovasculares similares: el primero y el tercero reanimado de una muerte súbita secundario a una taquicardia ventricular con compromiso hemodinámico, y el segundo, con episodios sincopales recurrentes. Todos con grados importantes de afectación miocárdica con deterioro severo de la función ventricular izquierda y con taquicardia ventricular inducible cuyo mecanismo es la reentrada entre las ramas eléctricas del sistema normal de conducción cardíaca. El primer y tercer caso con disociación VA evidente y el segundo caso con conducción VA 1:1 lo que produce dificultad diagnóstica adicional al incorporar otros diagnósticos diferenciales de taquicardia de QRS ancho con hisiograma precediendo la señal ventricular. Además, el primer paciente presentó en su seguimiento episodios recurrentes de taquicardia auricular sostenida y fibrilación auricular paroxística, lo que dificulta aún más el manejo y obliga a tomar medidas adicionales para evitar terapias inapropiadas por el desfibrilador.

La Distrofia miotónica de Steinert, también conocida como distrofia miotónica de tipo 1, es una enfermedad muscular caracterizada por miotonía y daño multiorgánico que combina diversos grados de debilidad mus- 
cular, arritmias y/o trastornos de conducción cardíaca, cataratas, daños endocrinos, trastornos del sueño y calvicie. Es la más frecuente de las distrofias miotónicas de aparición en la edad adulta y su prevalencia es variable; 1/1.000.000 habitantes en algunas áreas de Japón, 3-15/100.000 en Europa hasta 1/500 en Québec. Es más frecuente en hombres. La enfermedad está asociada con anomalías en el gen CMPK (Myotonic Dystrophy Protein Kinase), específicamente en el locus 19q13-2 (repetición anormalmente elevada del triplete CTG: el valor normal es de 5 a 37 repeticiones. Este gen codifica para una proteína quinasa expresada en el músculo esquelético, miotonina. Pacientes con 38 a 49 repeticiones son asintomáticos y las repeticiones varían entre 50 a 4000). La transmisión es autosómica dominante y puede ocurrir con anticipación, es decir, la enfermedad puede ser más grave y aparecer antes en la descendencia, debido al aumento en el número de repeticiones CTG heredadas en hijos de padres afectados. La detección de la anomalía 19q13-2 utilizando técnicas de genética molecular confirma el diagnóstico en un $100 \%$. El curso de la enfermedad consiste generalmente en un deterioro lento y progresivo, pero en ocasiones se ha observado una evolución rápida. La esperanza de vida se reduce por el aumento de la mortalidad asociada a las complicaciones pulmonares y cardíacas ${ }^{2}$.

Las alteraciones de la conducción y las taquiarritmias son comunes en las distrofias miotónicas y contribuyen a la morbimortalidad. Los pacientes pueden presentar muerte súbita, disfunción progresiva del ventrículo izquierdo, infarto de miocardio, taqui y bradiarritmias.

La relación entre la enfermedad de Steinert y la presencia de taquicardia ventricular rama- rama (TVRR) ha sido ampliamente descrita por varios autores y es la forma más frecuente de TV en este tipo de pacientes 3,4 . En nuestra serie de 3 pacientes, todos tenían un deterioro severo de la función ventricular izquierda al momento del diagnóstico de la arritmia ventricular (FEVI 30\%) y los tres habían sido reanimados de MS secundaria a TV. Esto difiere de la serie de Merino et al, en que 5 de los 6 pacientes presentados no tenían cardiopatía estructural evidente, a pesar de presentarse con episodios frecuentes de TV, lo que indica la falta de correlación entre el mecanismo de la taquicardia y la función ventricular izquierda4.

La TVRR es una arritmia cardíaca caracterizada por una macro reentrada dentro del sistema His - Purkinje. El circuito más común consiste en una conducción anterógrada a través de la rama derecha del haz de His seguida de conducción intramuscular transeptal y luego conducción retrógrada por alguno de los fascículos de la rama izquierda del His (Fig 8). Esto se manifiesta por una taquicardia regular con un patrón de bloqueo de rama izquierda en el ECG de superficie (Tipo 1) o de bloqueo de rama derecha (Tipo 2) cuando la dirección del circuito de propagación es en sentido opuesto5-7. Existe un tercer tipo, la TV interfascicular izquierda que está menos estudiada y con criterios diagnósticos menos establecidos8.

La TVRR se puede presentar con mayor frecuencia en pacientes con miocardiopatía dilatada, cirugía valvular aórtica y pacientes con distrofia muscular. En esta última se ha encontrado como el mecanismo responsable del $100 \%$ de todas las formas de TV sostenida y se considera el sustrato específico para el desarrollo de esta arritmia; sin embargo, también puede ocurrir en pacientes con cardiopatía isquémica e incluso en pacientes sin cardiopatía estructural9. La base fundamental de su mecanismo es la presencia de enfermedad sobre el sistema específico de conducción eléctrico cardíaco, con conducción lenta de una de las ramas, habitualmente la rama izquierda del haz de His.

El diagnóstico de TVRR se sospecha por la patología de base del paciente, el ECG y se establece mediante el estudio electrofisiológico. El diagnóstico diferencial se debe realizar entre las taquicardias regulares con QRS ancho y, en especial con morfología de BCRI, en el tipo 1: También es necesario diferenciarlas de TV de origen miocárdico y taquicardias supraventriculares conducidas con aberrancia, dentro de las cuales se deben descartar taquicardias ortodrómicas conducidas con aberrancia y antidrómicas mediadas por una vía accesoria atrio fascicular o atrioventricular con o sin comportamiento decremental (vías tipo Mahaim).

El ECG en ritmo sinusal y la comparación con el ECG en taquicardia es muy importante. En RS, con frecuencia se encuentra un trastorno de conducción de base con BAV de $1^{\circ}$ grado y BCRI. El ECG durante TVRR de tipo 1 muestra un patrón de BCRI con pequeñas diferencias de morfología del QRS respecto al observado en ritmo sinusal. Estas pequeñas diferencias de morfología en RS y TV se deben a que no existiría un bloqueo "completo" de la rama izquierda durante el RS, sino más bien un enlentecimiento de la conducción por esta rama, por lo que en RS el QRS es el resultado de una fusión entre el frente de activación de la rama derecha más alguna contribución de la rama izquierda; en cambio, el QRS durante la TVRR correspondería al frente de activación 
producido exclusivamente por la rama derecha.

Esta conducción lenta anterógrada y retrógrada de la rama izquierda del has de His corresponde al sustrato y la base fundamental para el mecanismo de este tipo de taquicardia.

Los principales criterios diagnósticos de TVRR se resumen en la Figura 8. En el estudio electrofisiológico observaremos con frecuencia un intervalo HV prolongado en RS. En TVRR clásica o tipo 1, a diferencia de las TV de origen miocárdico, la señal hisiana precede a la activación ventricular, con un intervalo $\mathrm{HV}$ en taquicardia $\geq \mathrm{HV}$ en RS, en paciente con BCRI de base. La señal de His siempre precede a la señal de rama derecha y al complejo QRS, a diferencia de la TV de origen miocárdico y las taquicardias antidrómicas por vías accesorias que utilizan el nodo AV en sentido retrógrado.

En los casos infrecuentes donde existe un patrón de BCRD en RS y TVRR tipo 1, es decir, conducción anterógrada por la rama derecha (rama lenta en este caso) y conducción retrógrada por la rama izquierda (rama rápida), el intervalo HV será negativo, sin embargo, lo que está traduciendo es un intervalo HV muy prolongado.

En las TVRR, con frecuencia observaremos oscilaciones en la longitud de ciclo, con cambios en el intervalo $\mathrm{H}-\mathrm{H}^{`}$ que preceden a los cambios del intervalo $\mathrm{V}-\mathrm{V}^{`}$. Este es el criterio clásico para el diagnóstico de TVRR y el que ha sido más utilizado en la práctica electrofisiológica, pero presenta ciertas limitaciones:

1) Pueden observarse variaciones en el intervalo $\mathrm{HV}$ por retraso en la conducción de la rama referente, por lo que no se cumpliría lo anterior, sino más bien las variaciones del intervalo $\mathrm{HH}^{`}$ seguirían en vez de preceder a las del intervalo VV; 2) Este criterio no permitiría distinguir la TVRR de la taquicardia por reentrada intranodal con trastorno de conducción intraventricular, especialmente cuando hay una relación VA 1:1 y en los raros casos de taquicardia intranodal con disociación ventrículo atrial; y 3). La limitación más importante, es que en muchos casos no se consigue un registro hisiano estable durante taquicardia, ya sea por movilidad del catéter que registra el His o por enfermedad severa de conducción, en donde estos potenciales son de muy baja amplitud como se presenta en uno de nuestros casos presentados.

Por estas razones se han creado criterios diagnósticos alternativos a los clásicos mediante el uso de maniobras de encarrilamiento (respuesta a la estimulación durante taquicardia, a un ciclo levemente inferior, con la finalidad de poner todos los componentes eléctricos de ésta a la nueva frecuencia, sin interrupción luego del cese de la estimulación) desde el ápex del ventrículo derecho, en cercanía del sistema normal de conducción, y desde la aurícula, que nos ayudarán en el diagnóstico diferencial con otras taquicardias de QRS ancho (ventriculares y no ventriculares) y de taquicardias con relación VA 1:1, diferenciándola de taquicardias supraventriculares:

1) En TVRR el encarrilamiento desde el ápex del VD mostrará un intervalo post estimulación igual o muy cercano $(<30 \mathrm{~ms})$ al ciclo de la taquicardia con mínimo grado de fusión o fusión oculta10. En uno de nuestros pacientes se observó fusión oculta debido a captura directa de la región distal de la rama derecha. Esto descartaría una taquicardia supraventricular conducida con aberrancia, por ejemplo, taquicardia intranodal con BCRI, pero no descartaría una taquicardia ortodrómica mediada por una vía accesoria septal, con conducción aberrante. En los casos sin conducción VA no existiría duda diagnóstica, pero en aquellos con relación VA $1: 1$, se requieren maniobras adicionales para demostrar una conducción retrógrada por una vía accesoria oculta como, por ejemplo, preexcitación auricular retrógrada con His refractario.

2). Encarrilamiento con fusión oculta ortodrómica y manifiesta durante estimulación auricular y ventricular derecha respectivamente. El encarrilamiento de la TV con estimulación auricular con fusión oculta ortodrómica (se mantiene el QRS de la taquicardia) descarta la reentrada miocárdica a favor de la TVRR11.

Esta condición que se da, principalmente, en individuos con enfermedad cardíaca estructural, se puede presentar también en sujetos sanos que presenten específicamente enfermedad a nivel del sistema His - Purkinje ${ }^{12}$, especialmente, en sujetos menores de 60 años en quienes se ha demostrado una etiología genética de la enferme$\mathrm{dad}^{13}$. Roberts et al estudiaron 6 pacientes con TVRR y ausencia de cardiopatía estructural, encontrando una mutación culpable en 3 de ellos: 2 en SCN5A (Ala1905Gly [novo] y c.4719C $>\mathrm{T}$ [splice site mutation]) and 1 en LMNA (Leu327Val [novo]). La mutación SCN5A (Ala1905Gly) produce una reducción significativa de la densidad de corriente máxima del canal de sodio dependiente de voltaje con una pérdida de función y la mutación SCN5A c.4719C >T ha sido previamente reportada como causante en algunos casos de síndrome de Brugada. Por otra parte, la mutación LMNA (Leu327Val) es causante del fenotipo clásico de laminopa- 
tía. Los autores hacen énfasis respecto a la posibilidad de curar esta causa de muerte súbita de origen genético mediante la ablación con catéter ${ }^{14}$.

\section{Ablación y resultados:}

La ablación con RF de este tipo de taquicardia es la terapia de elección en todos los pacientes y es relativamente fácil de realizar, con una elevada tasa de éxito. Consiste en interrumpir parte del circuito obligado de la taquicardia, ablacionando la rama derecha del haz de His. Esto se puede realizar durante taquicardia o en ritmo sinusal. Los pacientes pueden desarrollar BAVC intra o infrahisiano durante la ablación o en el seguimiento, dependiendo de la serie entre un 0 y $30 \%$, por lo que habitualmente requieren de un implante de marcapasos o desfibrilador dependiendo de la estratificación de riesgo en cada caso ${ }^{4,7,15-17}$.

El segundo paciente de nuestra serie, presentó un BAVC permanente producto de la ablación con RF. La posible explicación fue que no conseguimos visualizar con claridad el registro de His y en forma inadvertida realizamos una ablación muy proximal de la rama derecha. La forma de llevar a cabo la ablación consiste en identificar la señal de His y luego la señal de rama derecha (HV > RD-V) avanzando sutilmente el catéter de ablación en dirección apical, con tal de evitar el riesgo de lesión de His e inducción de BAVC. Los criterios clásicos son registro de potencial de rama derecha a lo menos 20 ms posterior al registro de His y ausencia de potencial auricular en el mismo canal.

Como alternativa a la ablación de la rama derecha, se ha propuesto la ablación de la rama izquierda del haz de His, sin embargo, es más dificultosa de realizar por su división más precoz ${ }^{18,19}$.

Más recientemente se ha descrito también la posibilidad de realizar una ablación del fascículo posterior de la rama izquierda del haz de His. En casos en que no se logre visualizar correctamente el potencial de rama derecha, la ablación guiada por anatomía no de resultado o presenta recurrencias intraprocedimiento o durante el seguimiento ${ }^{20}$.

La recurrencia de TV rama- rama luego de la ablación exitosa es muy poco probable. El seguimiento electrocardiográfico con persistencia de Bloqueo completo de rama derecha es un indicador de éxito a largo plazo. Pathak y col en una serie de 10 años (2005 al 2016) con 32 pacientes consecutivos ablacionados con RF en la rama derecha del His (59\%) y rama izquierda del His (41\%), no tuvieron recurrencia de TVRR en un seguimiento de alrededor de 8 años ${ }^{21}$.

Sin embargo, el pronóstico general de estos pacientes está determinado por la patología de base extracardíacas en los casos de distrofias miotónicas, o por el grado de compromiso de la función cardíaca. Los pacientes con cardiopatía estructural pueden presentar también arritmias ventriculares no relacionadas al sistema de conducción, sino a reentradas miocárdicas, lo que confiere un mayor riesgo de muerte súbita. En la serie de Pathak, la mortalidad global fue de $19 \%$. El $22 \%$ de los pacientes tenía intervalo HV normal y el $31 \%$ tenía función ventricular normal, no observándose mortalidad cardiovascular en estos subgrupos.

La indicación de implantar un desfibrilador con o sin resincronizador se ajusta a las recomendaciones clásicas para este tipo de pacientes. En nuestra serie, los tres pacientes tenían disfunción ventricular severa con una expectativa de vida mayor de un año, por lo que se optó por implantar un Desfibrilador. Si bien es discutible la necesidad de resincronización, más aun teniendo en cuenta la probabilidad de estimulación ventricular derecha que en las series llega al 30\%, ésta debe ser considerada dependiendo de las características generales y el estatus funcional de los pacientes. Los tres pacientes presentados estaban limitados funcionalmente por su patología neurológica de base, sin presentar síntomas de insuficiencia cardíaca, por lo que se optó por no resincronizar.

Un paciente falleció al cabo de 8 meses a causa de una neumonía aspirativa. En su seguimiento, no presentó nuevos episodios de arritmias ventriculares, al igual que en el seguimiento alejado de los otros dos pacientes. En uno de ellos, el dispositivo nos ayudó a identificar y tratar con éxito arritmias supraventriculares durante el seguimiento. 


\section{Bibliografía}

1. RODRIGUEZ TORRES D, JIMÉNEZ JAIMEZ J, MACÍAS RUIZ R, ÁLVAREZ LOPEZ M, TERCEDOR L. Manifestaciones cardíacas de las enfermedades neuromusculares. Rev Esp Cariol. 2018; 71(7): 580-594.

2. SCHOTTLAENDER. Las distrofias miotónicas: diagnóstico y manejo. Neurol Arg. 2010; 2(2):127-141.

3. KUZMICIC B, VALLS V, BRUGADA J, NAVARRO X, NAVARRO F. Taquicardia ventricular monomórfica sostenida en la distrofia miotónica. Rev Esp Cardiol. 1994; 47:843- 846.

4. MERINO JL, CARMONA JR, FERNÁNDEZ-LOZANO I, et al. Mechanisms of sustained ventricular tachycardia in myotonic dystrophy: implications for catheter ablation. Circulation. 1998; 98:541-546.

5. AKHTAR M, GILBERT C, WOLF FG, SCHMIDT DH. Reentry within the His-Purkinje system. Elucidation of reentrant circuit using right bundle branch and His bundle recordings. Circulation 1978;58: 295-304.

6. CACERES J, JAZAYERI M, MCKINNIE J, et al. Sustained bundle branch reentry as a mechanism of clinical tachycardia. Circulation 1989; 79:256-70.

7. BLANCK Z, DHALA A, DESHPANDE S, SRA J, JAZAYERI M, AKHTAR M. Bundle branch reentrant ventricular tachycardia: cumulative experience in 48 patients. J Cardiovasc Electrophysiol 1993;4: 253-62.

8. CRIJNS HJ, SMEETS JL, RODRIGUEZ LM, MEIJER A, WELLENS HJ. Cure of interfascicular reentrant ventricular tachycardia by ablation of the anterior fascicule of the left bundle branch. J Cardiovascular Electrophysiol 1995: 6(6): 486-492.

9. MERINO JL, PEINADO R, SOBRINO JA. Suden death in myotonic dystrophy: the potential role of bundle-branch reentry. Circulatio 2000; 101(5): E73.

10. MERINO JL, PEINADO R, FERNANDEZ-LOZANO I et al. Bundle branch reentry and the postpacing interval after entraintment by right ventricular ápex stimulation: a new approach to elucidate the mechanism of wide QRS complex tachycardia with atrioventricular dissociation. Circulation 2001; 103(8): 1102-1108

11. MERINO JL, PEINADO R, FERNANDEZ-LOZANO I, SOBRINO N, SOBRINO FA. Traansientt entraintment of bundle-branch reentry and ventricular stimulation: elucidation of the tachycardia mechanism through analysis of the surface ECG. Circulation 1999; 100 (17): 1784-1790.
12. BLANCK Z, JAZAYERI M, DHALA A, DESHPANDE S, SRA J, AKHTAR M. Bundle branch reentry: a mechanism of ventricular tachycardia in the absence of myocardial or valvular dysfunction. J Am Coll Cardiol 1993; 22:1718-22.

13. Baruteau A-E, Probst V, Abriel H. Inherited progressive cardiac conduction disorders. Curr Opin Cardiol 2015; 30:33-9.

14. ROBERTS J, GOLLB M, YOUNG CH, CONNORS S, GRAY $\mathrm{CH}$, WILTON S et al. Bundle Branch Re- Entrant Ventricular Tachycardia. Novel genetic mechanisms in a life -threatening arrhythmia. J Am Coll Cardiol EP 2017; 3: 276-88.

15. COHEN TJ, CHIEN WW, LURIE KG, YOUNG C, GOLDBERG HR, WANG YS, LANGBERG JJ, LESH MD, LEE MA, GRIFFIN JC, SCHEINMAN MM. Radiofrequency catheter ablation for treatment of bundle branch reentrant ventricular tachycardia: Results and long-term follow-up. J Am Coll Cardiol 1991; $18: 1767-1773$.

16. MEHDIRAD AA, KEIM S, RIST K, TCHOU P. Long-term clinical outcome of right bundle branch radiofrequency catheter ablation for treatment of bundle branch reentrant ventricular tachycardia. Pacing Clin Electrophysiol 1995; 18:2135-2143.

17. NARASIMHAN C, JAZAYERI M, SRA J, DHALA A, DESHPANDE S, BIEHL M, AKHTAR M, BLANK Z. Ventricular tachycardia in valvular heart disease: Facilitation of bundle-branch reentry by valve surgery. Circulation 1997; 96:43074313.

18. BLANK Z, DESHPANDE S, JAZAYERI MR, AKHTAR M. CATHETER ablation of the left bundle branch for the treatment of sustained bundle branch reentrant ventricular tachycardia. J Cardiovasc Electrophysiol 1995; 6:40-43.

19. SCHMIDT B, TANG M, CHUN KR, et al. Left bundle branchPurkinje system in patients with bundle branch reentrant tachycardia: lessons from catheter ablation and electroanatomic mapping. Heart Rhythm 2009; 6: 51-8.

20. SANTORO F, HAYASHI K, KUCK K, OUYANG F. Catheter Ablation of the Left Posterior Fascicle for Bundle Branch Reentrant Ventricular Tachycardia with Right Bundle Branch Block Morphology. A Stepwise Approach. JACC Clin Electrophysiol. 2017 Apr;3(4):415-416.

21. PATHAK RK, FAHED J, SANTANGELI P, HYMAN MC, LIANG JJ, KUBALA M et al. Long-Term Outcome of Catheter Ablation for Treatment of Bundle Branch Re-Entrant Tachycardia. JAAC Clin Electrophysiol 2018 Mar;4(3):331-338. 